\title{
RF Control Optimization and Automation for Normal Conducting Linear Accelerators
}

\author{
Zheqiao Geng, Member, IEEE
}

\begin{abstract}
Normal conducting linear accelerators are widely used in many Free Electron Laser (FEL) machines all over the world. They normally consist of multiple RF stations either with standing wave cavities (e.g. RF Gun) or with travelling wave accelerating structures working at different frequencies. Low Level Radio Frequency (LLRF) systems are used to measure the RF fields in cavities or structures and correct the fluctuations in RF fields with pulse-to-pulse feedback controllers. To facilitate the operations of multiple RF stations, the LLRF system should also provide algorithms and procedures to automate the setup, calibration and optimization of the RF systems. In this paper, several typical algorithms and procedures will be described, such as calibrating the DAC offset to reduce the RF leakage from vector modulator, calibrating the RF signal group delay and flattening the intra-pulse phase distribution with adaptive feed forward. The algorithms and procedures have been implemented as a LLRF High Level Applications (HLA) software for SwissFEL, which is an FEL machine under commissioning at Paul Scherrer Institute (PSI), Switzerland. The architecture of the LLRF HLA software will be introduced and the test results at SwissFEL will be also described.
\end{abstract}

Index Terms-LLRF, Normal Conducting Linac, High Level Applications, Automation

\section{INTRODUCTION}

$\mathrm{N}$ ormal conducting linear accelerators are widely used in FEL machines like the Linac Coherent Light Source (LCLS) [1] at the SLAC National Accelerator Laboratory and the SwissFEL [2] at PSI. In such machines, normally an RF Gun with standing wave cavity is used to generate electron bunches and multiple RF stations with travelling wave accelerating structures are used to accelerate the beam to desired energy [3-4]. The RF pulse length is normally within several microseconds and the pulse repetition rate is from tens to several hundred $\mathrm{Hz}$ (e.g. SwissFEL works at a repetition rate of $100 \mathrm{~Hz}$ with an RF pulse length up to $3 \mu \mathrm{s}$ ).

Digital LLRF systems are widely used to control the RF stations [5-6]. To detect the RF amplitude and phase, the RF frequency signals are down converted to Intermediate Frequency (IF) signals and then sampled by fast Analog-to-digital Converters (ADC) [7]. Field-Programmable Gate Arrays (FPGA) are usually used to process the ADC

Manuscript received May 14, 2017.

Z. Geng is with the RF Section, Department of Large Research Facilities (GFA), Paul Scherrer Institut, 5232 Villigen PSI, Switzerland (phone: +41 78 86380 59; e-mail: zheqiao.geng@psi.ch). samplings with digital demodulation algorithms [8-9]. For an RF station with multiple cavities powered by the same klystron, the vector sum of the RF signals from all cavities should be used to represent the integral RF field used for beam acceleration. Feedback controllers can be implemented in FPGAs [5] if fast feedback loops are needed (e.g. intra-pulse feedback with loop latency smaller than $1 \mu \mathrm{s})$. For the SwissFEL LLRF system, only pulse-to-pulse feedback is required and it is implemented in CPU [6] for better flexibilities. The control actuation from the LLRF system is usually applied to the RF station via a vector modulator driven by a pair of Digital-to-analog Converters (DAC). Normally the DACs generate base-band pulses to determine the pulse shape, amplitude and phase of the klystron output power. A typical RF station with a pulse compressor and four traveling wave structures controlled by the LLRF system is shown in Fig. 1. The output of vector modulator is amplified by a pre-amplifier and then used to drive a klystron, which further amplifies the RF power to tens of megawatts. Pulse compressors, such as the SLAC Energy Doubler (SLED) [10] and the Barrel Open Cavity (BOC) [11], are used in certain RF stations to further increase the peak RF power in the accelerating structures. The RF pulse length should be larger than the filling time of accelerating structures so that the electromagnetic field in the structures can be well established before injecting the beam.

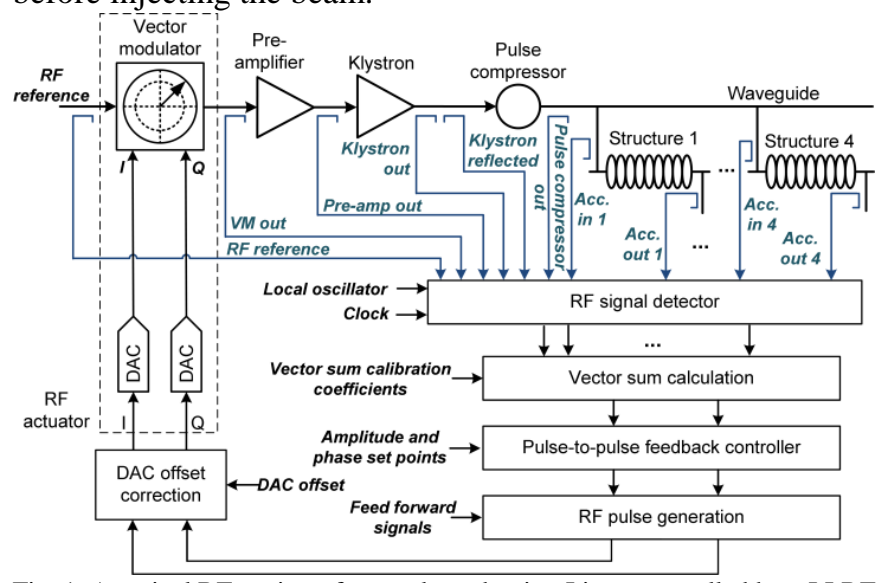

Fig. 1. A typical RF station of normal conducting Linac controlled by a LLRF system which is described as function blocks.

The LLRF system provides the following functions:

1) Provides precise and accurate phase and amplitude measurements of RF signals at various locations of the RF system.

2) Suppresses the RF field fluctuations in cavities and 
accelerating structures.

3) Facilitates the operators to easily setup and operate the RF stations.

In this paper, the third point will be focused on which is very important for the overall success of the LLRF system for good operability, understandability and reliability. The tools to facilitate the operations of RF stations are compiled in a software package named LLRF HLA. Fig. 2 shows the context of the LLRF HLA within the RF system.

The LLRF HLA takes mainly the RF measurements provided by the LLRF low level blocks (e.g. firmware and data acquisition software), executes certain procedures for calibration and optimization, and then sets the necessary parameters of RF system. For an RF station as Fig. 1, the typical algorithms and procedures are summarized in Table I.

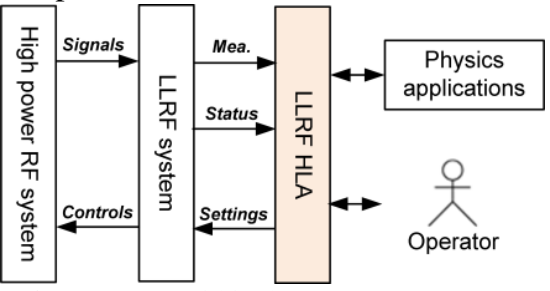

Fig. 2. Context of LLRF HLA within the RF system.

TABLE I

SUMMARY OF TYPICAL LLRF HIGH LEVEL APPLICATIONS

\begin{tabular}{cl}
\hline \hline \multicolumn{1}{c}{ Category } & \multicolumn{1}{c}{ HLA algorithm and procedure } \\
\hline RF setup & $\begin{array}{l}\text { Pulse shape optimization. } \\
\text { DAC offset correction. }\end{array}$ \\
& Klystron working point determination [12]. \\
Group delay calibration. & Loop gain and loop phase calibration. \\
Accelerating voltage and beam phase calibration [13]. & \\
Optimization & RF pulse flattening. \\
& Feedback gain optimization [14]. \\
Cavity detuning measurement [15]. & Structure detuning measurement. \\
\hline \hline
\end{tabular}

Some of the algorithms and procedures will be described in details in the next section.

\section{LLRF HLA ALGORITHMS AND PROCEDURES}

\section{A. Pulse Shape Optimization}

The basic shape of the RF pulses is determined by the DAC output signal from LLRF system. Most RF stations need a square pulse in amplitude and a constant phase except for the ones with pulse compressors, which require a phase jump by $180^{\circ}$ after a filling period of the compressor cavities [10-11].

The RF Gun is normally a multi-cell standing wave cavity with different pass-band modes for the basic TM010 mode. As an example, the SwissFEL RF Gun [4] is a 2.6-cell standing wave cavity working at $2998.8 \mathrm{MHz}$ ( $\pi$ mode). The nearest pass-band mode ( $\pi / 2$ mode) is about $16 \mathrm{MHz}$ away in spectrum. A sharp square pulse generated by DAC results in a wide band in the klystron output which stimulates the $\pi / 2$ mode significantly. The pass-band mode signals will corrupt the RF field measurement and generate problems in closed loop operation. In order to reduce the bandwidth of the Gun drive power, the rising and falling edges of the RF pulse was smoothened with different patterns like linear ramping and half sine signal (see Fig. 3). Experiments showed that the half sine signal could effectively suppress the pass-band modes in the Gun cavity which can be seen in Fig. 4 , where the $\pi / 2$ mode signal was aliased to around $58 \mathrm{MHz}$.

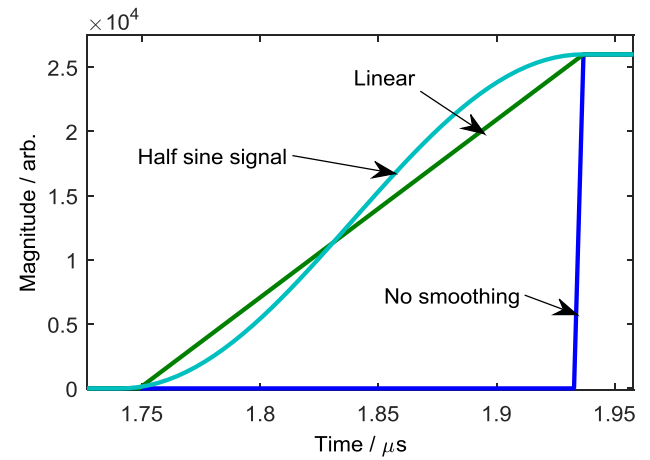

Fig. 3. Strategies to smoothen the rising edge of DAC pulse.
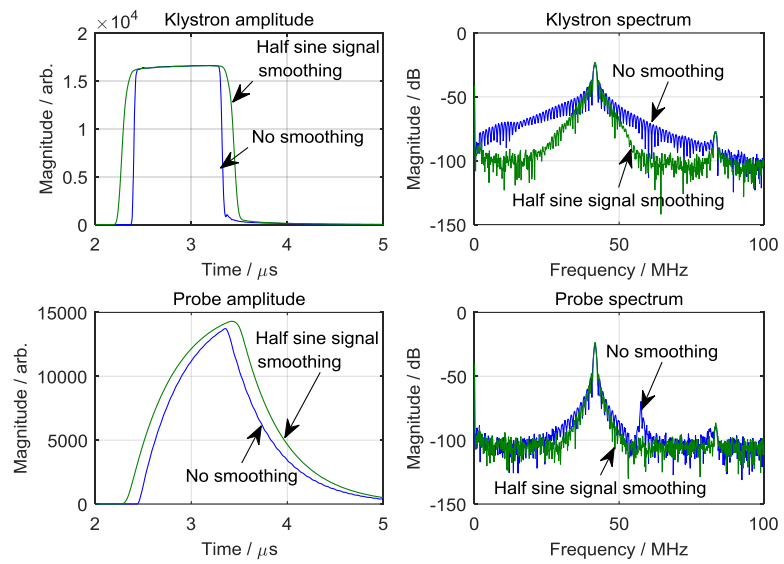

Fig. 4. Amplitude waveforms and spectrums of klystron output and cavity probe signals. The spectrums were calculated with the ADC samplings of the down converted IF signals at $41.65 \mathrm{MHz}$. The sampling frequency of the DAC and $\mathrm{ADC}$ was $249.9 \mathrm{MHz}$.

\section{B. DAC Offset Correction}

The most widely used strategy for the RF actuator in LLRF system consists of one vector modulator and two DACs (see Fig. 5). The two DACs are used to adjust the amplitude and phase of the RF reference signal input to the vector modulator. Due to the imperfection of the vector modulator, there is always RF reference leakage to the output port even the DACs have zero outputs. The leakage will generate significant errors in phase and amplitude actuations [5]. To compensate the RF leakage, constant offsets are added in the DAC signals to generate an extra RF output with the same amplitude but opposite phase with respect to the RF leakage signal, resulting in the cancellation of the leakage. 


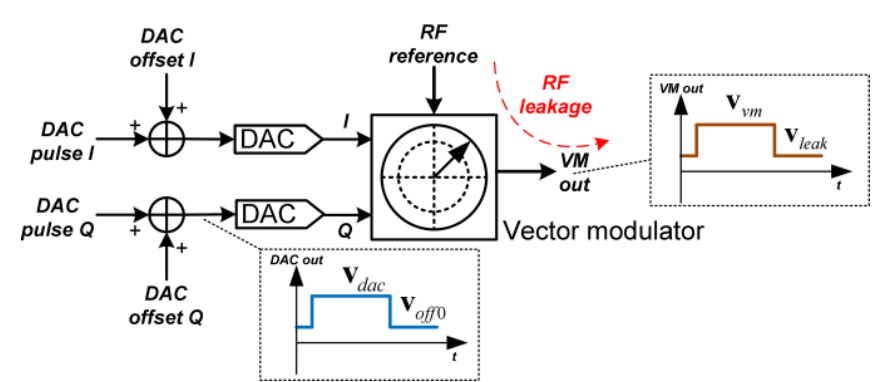

Fig. 5. Block diagram of the RF actuator for LLRF system.

To determine the DAC offset, a test pulse can be generated by the DACs, then the DAC output waveforms can be recorded and the RF signal of the output of vector modulator can be measured with the RF detector. Both signals are in in-phase (I) and quadrature $(\mathrm{Q})$ formats [16] corresponding to the real and imaginary parts of the complex signals. From the DAC waveform, the complex values of DAC initial offset $\left(\mathbf{v}_{\text {off }}\right)$ and DAC pulse $\left(\mathbf{v}_{d a c}\right)$ can be calculated. Similarly, the RF leakage $\left(\mathbf{v}_{\text {leak }}\right)$ and vector modulator output pulse $\left(\mathbf{v}_{\mathrm{vm}}\right)$ can be identified from the measurement of the vector modulator output signal. The complex gain between the DAC output and the vector modulator output can be defined as

$$
\mathbf{G}=\frac{\mathbf{v}_{v m}-\mathbf{v}_{\text {leak }}}{\mathbf{v}_{d a c}-\mathbf{v}_{\text {off } 0}} .
$$

Then in order to compensate the RF leakage, the DAC offset should be changed to

$$
\mathbf{v}_{\text {off }}=\mathbf{v}_{\text {off } 0}-\mathbf{G}^{-1} \mathbf{v}_{\text {leak }} \text {. }
$$

In the calculation, the initial DAC offset was taken into account so that the algorithm can be executed iteratively. The algorithm was successfully implemented in the SwissFEL LLRF system and the results are shown in Fig. 6. The RF leakage can be well compensated with one or two iterations of correction.
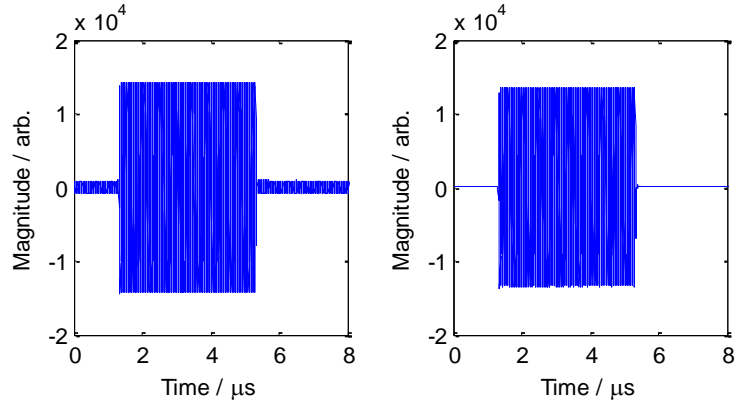

Fig. 6. Intermediate frequency signal of the vector modulator output without (left) and with (right) DAC offset correction.

\section{Group Delay Calibration}

In LLRF system, DACs initialize the RF pulse and the RF power is amplified and transmitted to the cavities or structures for beam acceleration. The LLRF system also picks up the RF signals from different locations of the RF system with usually random cable length. The uncertainties in both RF driving paths and measurement paths generate different group delays in the measured RF signals. In order to compare the relative RF errors in different locations of the RF system, the corresponding parts of the RF pulses need to be used. To suppress the intra-pulse ripple at a certain point within the RF pulse, we also need to know which DAC point before needs to be adjusted. These all need an accurate calibration of the group delays between the measurements of RF signals and the DAC output.

To calibrate the group delays, a single point in the DAC pulse can be varied (e.g. with smaller magnitude) as a bump in the pulse to generate a transient in all RF signals. The overall path converting the DAC bump to the measured transient of an RF signal can be viewed as cascaded low-pass filters contributed from different components in the signal path. The transfer function of the signal path can be described as

$$
H(s)=\frac{\prod_{n=1}^{N} k_{n}}{\prod_{n=1}^{N}\left(\tau_{n} s+1\right)},
$$

where $k_{n}$ is the gain and $\tau_{n}$ is the time constant of the $n$th component in the signal path, $N$ is the total number of components. The cascaded filters in (3) will expand the DAC bump pulse and also generate extra group delays in addition to the delays introduced by RF transmission lines. The overall group delay of an RF signal can be estimated by comparing the time difference between the DAC bump and the transient in the RF signal. A simulation was carried out to study the algorithm by calculating the transient responses of three cascaded low-pass filters for a $4 \mathrm{~ns}$-width input bump. It was assumed that the three filters were identical with a bandwidth of $10 \mathrm{MHz}$ and the group delay introduce by each of them was $10 \mathrm{~ns}$. The simulation results are shown in Fig. 7, from which the following information can be derived with the $3^{\text {rd }}$ filter as example:

1) The group delay between the DAC and the filter output can be estimated by comparing $t_{1}$ and $t_{0}$.

2) The time delay to achieve the maximum effect on the filter output by an adjustment on DAC can be estimated by comparing $t_{2}$ and $t_{0}$.

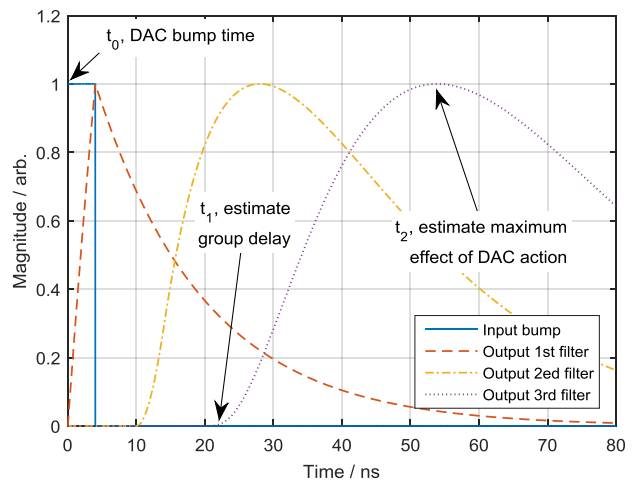

Fig. 7. Simulation of the algorithm for group delay calibration.

The algorithm has been applied to the routine setup of the SwissFEL RF stations. As an example, Fig. 8 shows the results of the group delay calibration between the DAC output and the vector modulator output of a SwissFEL RF station. 

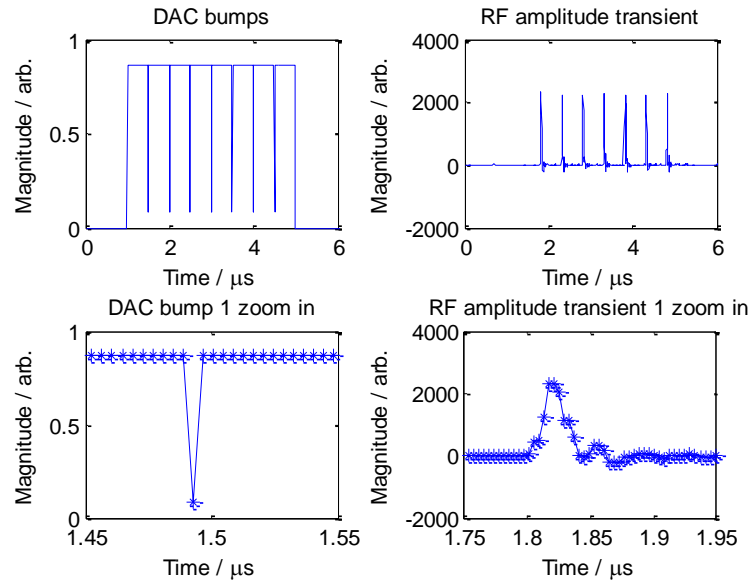

Fig. 8. Group delay calibration. Multiple bumps were applied to improve the resolution. The absolute value of the transient has been used. The calculated group delay is $0.308 \mu \mathrm{s}$ and the time delay for maximum effect from DAC action is $0.324 \mu \mathrm{s}$. The clock frequency for the DAC and the RF detector ADC was $249.9 \mathrm{MHz}$.

\section{Loop Gain and Loop Phase Calibration}

The RF pulses of normal conducting Linacs are normally too short for intra-pulse feedbacks. Pulse-to-pulse feedback loops are used to reduce the slow fluctuations in the RF fields. A general architecture of the amplitude and phase feedback loops is depicted in Fig. 9.

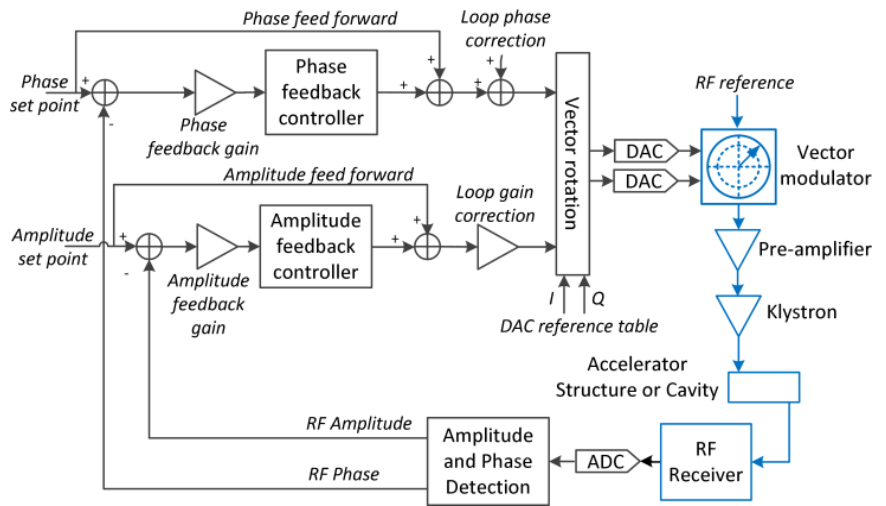

Fig. 9. Architecture of pulse-to-pulse feedback loops.

The RF field in the accelerating structure or cavity is measured with an RF receiver and digitized by an ADC. Then the amplitude and phase of the RF field are calculated with digital demodulation algorithms (e.g. non-IQ demodulation) [8-9]. Two feedback loops are applied on amplitude and phase respectively. Compared to the feedbacks on I and Q components of the RF signals [17], the feedbacks on amplitude and phase provide more flexibilities allowing the klystron to work in saturation but still with phase feedback applied. Feed forward controls are also applied to speed up the responses of the feedback loops and to provide a way to adjust the amplitude and phase of the RF station in open loop.

The feedback loops should be stable at different working points of the klystron which is typically non-linear (see Fig. 10). The system gain of the amplitude loop is defined as the incremental gain of the system at the working point between the output and input amplitudes, which can be calculated as the ratio between the variations in the system output and input with respect to the working point. Normally the variations should be small so that the system can be viewed approximately as a linear system around the working point. The system gain should be well calibrated so that the feedback gain can be determined. With a discrete integral feedback controller as described in reference [5], the maximum loop gain, which is the product of the feedback gain and the system gain, should be smaller than 2 to keep the loop stable. At a certain klystron working point as in Fig. 10 (b), the system gain can be calculated with the slope of the klystron gain curve at the working point.
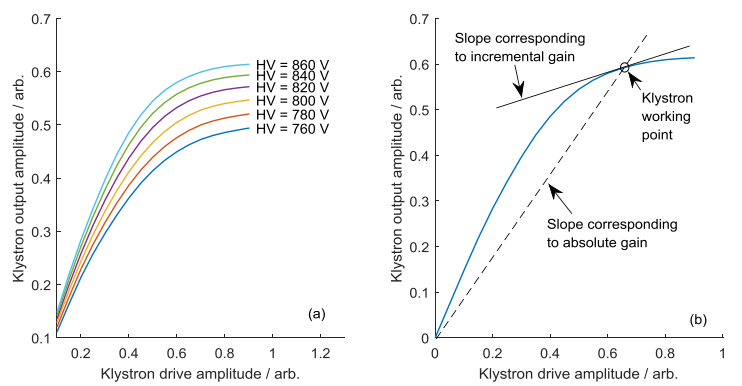

Fig. 10. Klystron non-linearity. (a) Klystron gain curves with different modulator high voltages (HV); (b) Absolute and incremental gains.

The system gain of the amplitude loop needs to be calibrated during run-time whenever the klystron working point changes. To do this, a small step is introduced at the end of the RF pulse with the timing chosen to avoid influencing the beam. The duration of the step is set to be as small as possible not to change the heat load of the RF structures. Fig. 11 shows a test in a working RF station with the pulses of the system input (DAC output) and the system output (klystron output). The system gain can be estimated by three different magnitudes of the small step at the end of the RF pulse.
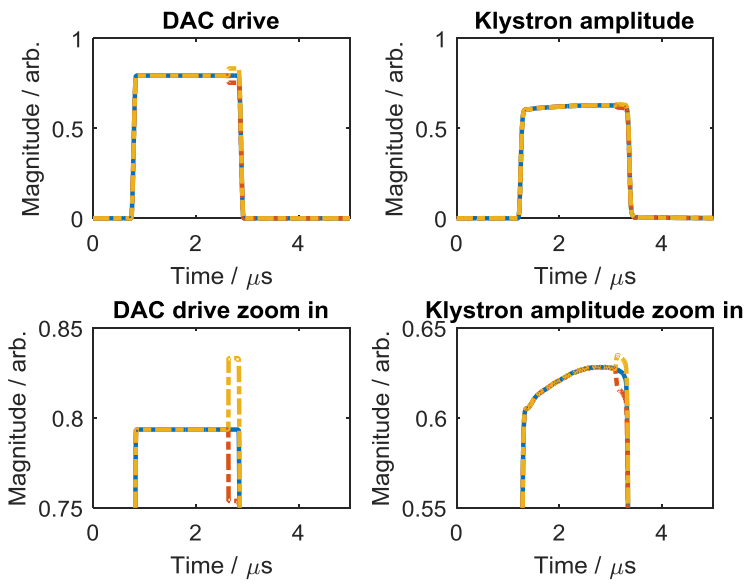

Fig. 11. Run-time calibration of the system gain (incremental gain) of the amplitude feedback loop. The system input amplitude (DAC output) was changed by $5 \%$ in positive and negative directions, and the system output amplitude (klystron output) changed by $1.3 \%$ and $-1.8 \%$ respectively. The system gain was estimated as 0.31 at the working point.

With the loop configuration in Fig. 9, we also would like the amplitude measurement equals to the set point in open loop with only feed forward control. This helps to have relatively accurate amplitude setting even the feedback loop is open, especially in the linear region of the klystron gain curve. In order to do this, 
the absolute gain as in Fig. 10 (b) should be also calibrated. With these considerations, the amplitude loop can be calibrated for a new klystron working point with the following two steps:

1) Calibrate the absolute gain so that the amplitude measurement equals to the set point in open loop.

2) Calibrate the system gain and determine the feedback gain to achieve the desired loop gain which is determined by the expected closed-loop dynamics and performances.

For most RF stations in SwissFEL, the amplitude feedback loops are mainly used to reduce the long-term drifts, and the loop gain is normally set low (e.g. 0.1 ) to avoid increasing the pulse-to-pulse jitters [5]. Fast changing of the amplitude set point is usually done in open loop with feed forward control. The major benefit of the on-line system gain calibration is to avoid the feedback loop entering the over-saturation region of the klystron gain curve, where the feedback loop becomes unstable due to the sign flip of the incremental gain.

Several klystron linearization methods [18-19] have been developed to overcome the non-linearity issues, which are mostly used for the RF stations equipped with intra-pulse feedbacks (e.g. RF stations for superconducting cavities).

The system gain for the phase loop is always 1 because any phase change at the input will cause exactly the same phase change at the output. And in order to have the ability for accurate open-loop phase adjustment, the loop phase should be calibrate so that the phase measurement equals to the phase set point even in open loop with the feed forward control.

\section{E. RF Pulse Flattening}

A flat RF pulse in both amplitude and phase are important for multi-bunch operation because all bunches require equal acceleration in the same RF pulse. Multiple iterative learning control (ILC) [20-21] algorithms have been developed to achieve flat amplitude and phase within the RF pulse. They are relatively complicated usually with complex optimization algorithms involved. In practice, if the response of the RF system is fast, a simpler algorithm can be used for which the errors in the output pulse can be applied to the DAC drive in a manner similar as the discrete integral feedback, see Fig. 12.

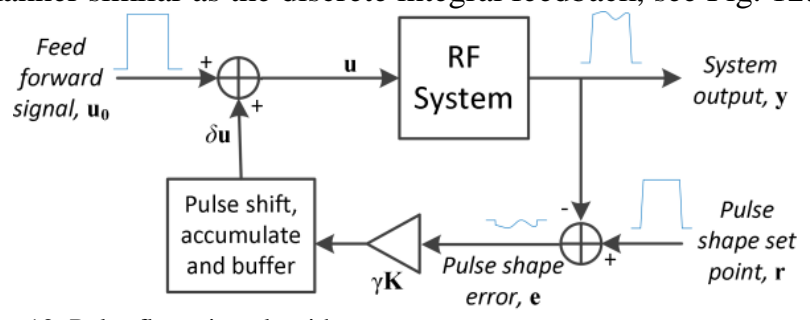

Fig. 12. Pulse flattening algorithm.

Assume the measurement of the $k$ th pulse at the system output is $\mathbf{y}(k, n)$ with $n$ the sample index in the pulse, and the pulse shape set point is $\mathbf{r}(n)$, the algorithm below can be used to determine the feed forward correction signal at the DAC output for the next pulse:

$$
\begin{aligned}
& \mathbf{e}(k, n)=\mathbf{r}(n)-\mathbf{y}(k, n) \\
& \delta \mathbf{u}(k+1, n-d)=\delta \mathbf{u}(k, n-d)+\gamma \mathbf{K} \cdot \mathbf{e}(k, n) . \\
& \mathbf{u}(k+1, n)=\mathbf{u}_{0}(n)+\delta \mathbf{u}(k+1, n)
\end{aligned}
$$

The term $\mathbf{e}(k, n)$ is the error of each sample in the $k$ th pulse. $\mathbf{K}$ is a complex number to calibrate the arbitrary phase difference and attenuation between the DAC and the location (e.g. klystron output) where the RF signal is picked up. $\gamma$ is the gain which should be normally between 0 and 1 . Then the DAC correction $\delta \mathbf{u}$ for the next pulse can be calculated recursively. The term $d$ in the index of DAC correction pulse is used to compensate the time delay between the DAC output and the pickup location of the output signal. The delay has been calibrated with the algorithm described before and it helps to locate the DAC point which should be corrected to compensate the errors in the corresponding point of the output signal.

The accuracy of delay $d$ is critical for the stability of the ILC algorithm. The error in $d$ will apply the feed forward correction signal on the wrong place of the DAC table, resulting in extra errors in the system output. Normally the accuracy of $d$ should be better than one or two DAC samples.

Compared to the feedback loops in Fig. 9, the ILC algorithm in equation (4) is equivalent to applying the pulse-to-pulse integral feedback on each point of the waveform. The stability criteria of the discrete integral feedback loop also applies to the gain $\gamma$ in equation (4), which should be smaller than 2 theoretically. In the implementation of the ILC algorithm for SwissFEL, the first several iterations use a larger gain (e.g. 1) for fast convergence and the remaining iterations use a smaller gain (e.g. 0.1) to reduce the high frequency noises.

In equation (4), the dynamics in the system are neglected, so it only works for fast systems with a time constant comparable to the clock cycle of the DACs and ADCs used in the LLRF system (e.g. the clock cycle for SwissFEL LLRF system is around 4 ns). For a system with a large time constant (e.g. systems with standing wave cavities with high quality factors), the algorithm in equation (4) can generate big oscillations spreading from the beginning of the RF pulse. Fig. 13 shows an example by applying the ILC algorithm to a cavity with a half-bandwidth of $3 \mathrm{MHz}$ running at $2998.8 \mathrm{MHz}$. The set point of the amplitude waveform of the RF signal was a square function, which is in principle not achievable due to the dynamics of the RF system, but it can be used to study the limitations of the ILC algorithm. From equation (4), the DAC correction signal is generated by simply scaling the errors of the output signal with respect to the set point. And due to the dynamics of the system, the errors at the beginning of the output pulse will not be fully compensated and it will be added to the DAC correction signal in every iteration, resulting in oscillations in the output signal.
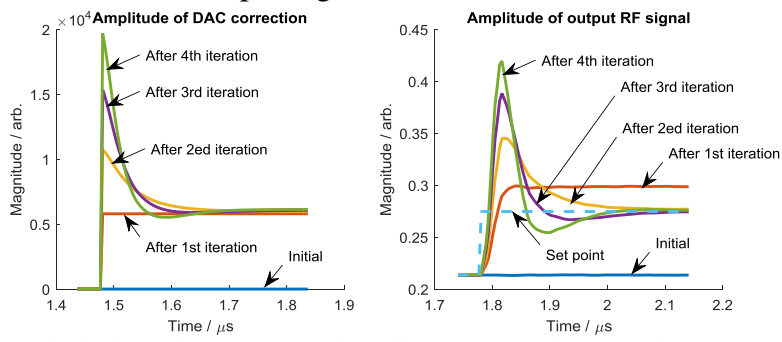

Fig. 13. Limitation of the ILC algorithm for a system with large time constant.

Though with the limitations described above, the algorithm is 
still practical for the systems with the following conditions:

1) The system has a fast response to the input. For a stepping input, the system should reach steady state within a period no longer than several clock cycles.

2) The ILC is used to flatten the small ripples in the pulse instead of generating large steps as in Fig. 13.

3) The ILC can be activated for a short time to generate flat pulses after the working point of the RF system is changed, without requiring continuous running of the ILC.

In the implementation for SwissFEL, the DAC correction signals are limited to reduce the oscillations as in Fig. 13.

The algorithm was tested in the SwissFEL C-band (5712 $\mathrm{MHz}$ ) test stand on the output of the klystron (with a half-bandwidth larger than $5 \mathrm{MHz}$ ) and the results are shown in Fig. 14. The pulse region of interests was flattened with the ILC algorithm and it converged within 5 iterations to achieve intra-pulse RMS fluctuations close to the resolution of the RF detector.

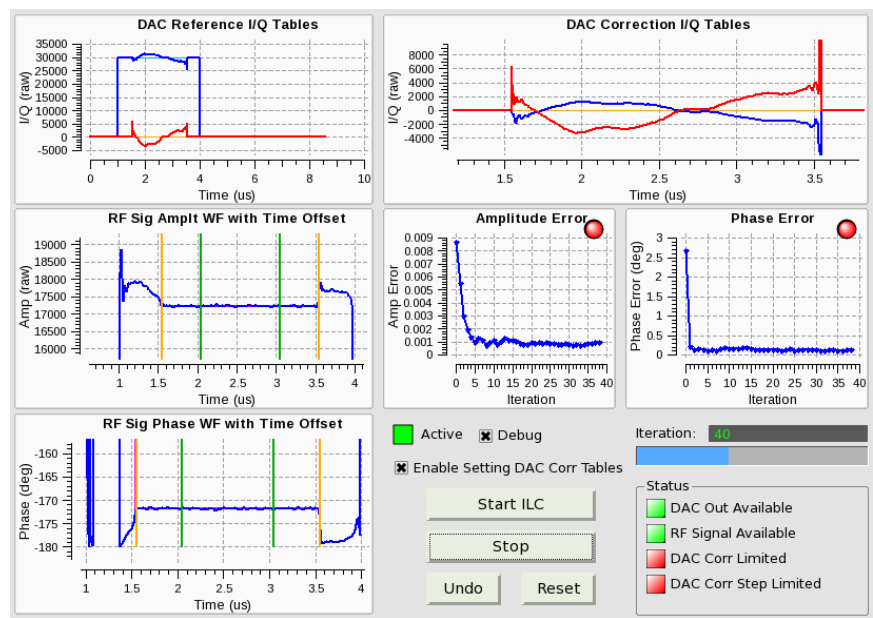

Fig. 14. Application of the pulse flattening algorithm on both amplitude and phase of the C-band klystron output. The correction gain $\gamma$ was set to 1 for the first 5 iterations and 0.1 for the remaining iterations.

\section{F. Structure Detuning Measurement}

Temperature fluctuations of the normal conducting cavities or travelling wave accelerating structures will generate detunings resulting in amplitude and phase errors in the RF fields. On the other hand, if we can measure the detuning in cavities and structures, the temperature set point in the cooling system can be adjusted to compensate the detuning. This is helpful to speed up the response of the cooling system especially when the RF power load in the cavities or structures changes much (e.g. RF power recovery after a klystron interlock trip).

The detuning of standing cavities can be measured by the phase slope of probe signals after the drive power vanishes [15]. The detuning of the travelling wave structures can be measured by comparing the phases between the output and input RF signals (mark the phase difference as $\Delta \varphi$ ). Normally when the structure is well tuned, which can be done by maximizing the beam acceleration, the phase difference can be recorded as a reference $\Delta \varphi_{0}$, and then the detuning of the structure will be proportional to the variations of the phase difference between the output and input signals represented as $\Delta \omega \sim \Delta \varphi-\Delta \varphi_{0}$.

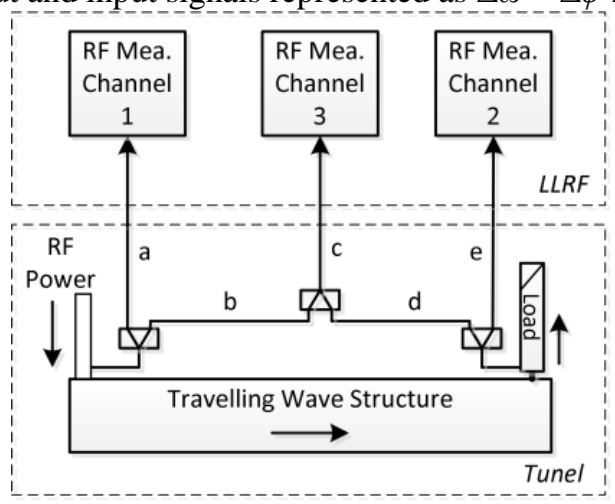

Fig. 15. Detuning measurement strategy for travelling wave accelerating structure.

In order to reduce the uncertainties caused by the cable drifts, it is better to measure the structure input and output signals with the same cable and the same RF detector, see Fig. 15. The input and output signals of the structure are combined with short but symmetric cables in the tunnel and then measured with a separate RF detector channel. The cables $b$ and $d$ are much shorter (around $1 \mathrm{~m}$ ) than the common cable c (longer than 10 $\mathrm{m}$ ), and because they experience the similar temperature changes, their phase drifts will be mostly canceled out when comparing the phases of the two RF signals delivered by them. An algorithm was developed to retrieve the input and output RF signals from the sampling of the combined RF signal (channel 3 ) and the structure input RF signal (channel 1). Then the phase difference of the retrieved signals is used to estimate the detuning of the structure. Fig. 16 shows the measurements of the structure input signal and the combined signal (top). Due to the group delay of the travelling wave structure, which is also known as the filling time $\left(T_{f}\right)$ of the structure, the first part of the combined signal within $T_{f}$ is only contributed from the structure input signal with a complex factor caused by the arbitrary attenuations and phase shifts in the measurement channels.
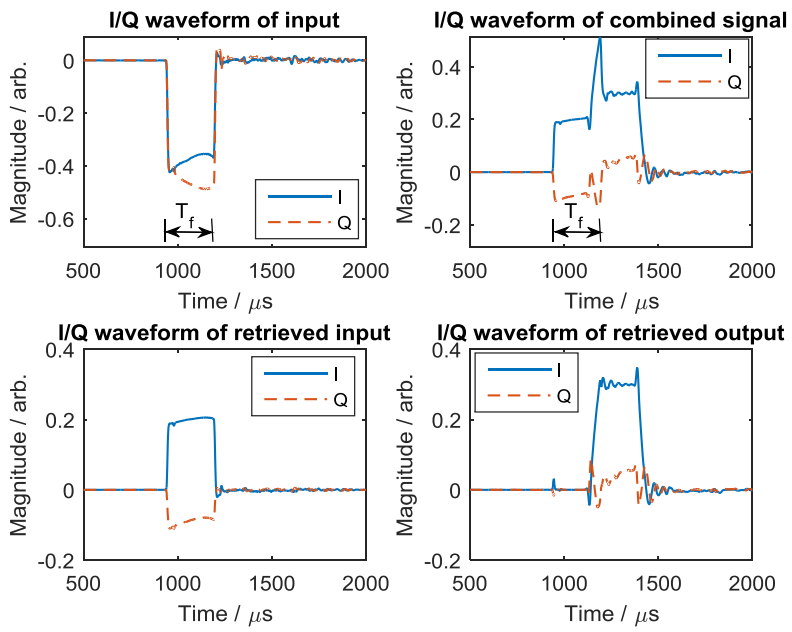

Fig. 16. Algorithm to retrieve the input and output signals from the combined signal. In this measurement, the pulse width was similar to the filling time of the structure. Normally the pulse width can be much bigger than $T_{f}$.

The complex factor (labeled as $\mathbf{C}$ ) can be calibrated with the waveforms of the structure input signal and the combined signal 
within the filling time of the structure. Then in the combined signal, the contribution from the structure input signal can be estimated from the channel 1 measurement and the factor $\mathbf{C}$, and by subtracting the input contribution from the combined signal, the contribution from the structure output signal can be calculated. The algorithm is described as:

$$
\begin{aligned}
& \mathbf{C}=\int_{0}^{T_{f}} \mathbf{v}_{\text {in }}(t) d t / \int_{0}^{T_{f}} \mathbf{v}_{c m b}(t) d t, \\
& \mathbf{v}_{\text {in_retrieve }}=\mathbf{C} \cdot \mathbf{v}_{\text {in }}, \\
& \mathbf{v}_{\text {out_retrieve }}=\mathbf{v}_{c m b}-\mathbf{v}_{\text {in_retrieve }}
\end{aligned},
$$

where $\mathbf{v}_{i n}$ and $\mathbf{v}_{c m b}$ are the I/Q waveforms of the structure input signal and the combined signal, while $\mathbf{v}_{\text {in_retrieve }}$ and $\mathbf{v}_{\text {out_retrieve }}$ are the retrieved structure input and output signals from the combined signal (see Fig. 16 bottom). Fig. 17 shows the phases of the retrieved structure input and output signals and the temperature of the structure measured at one of the S-band (2998.8 MHz) RF station of SwissFEL. The phase difference between the structure output and input signals showed linear correlation with the temperature and the phase-difference-temperature coefficient can be calibrated with linear fitting.
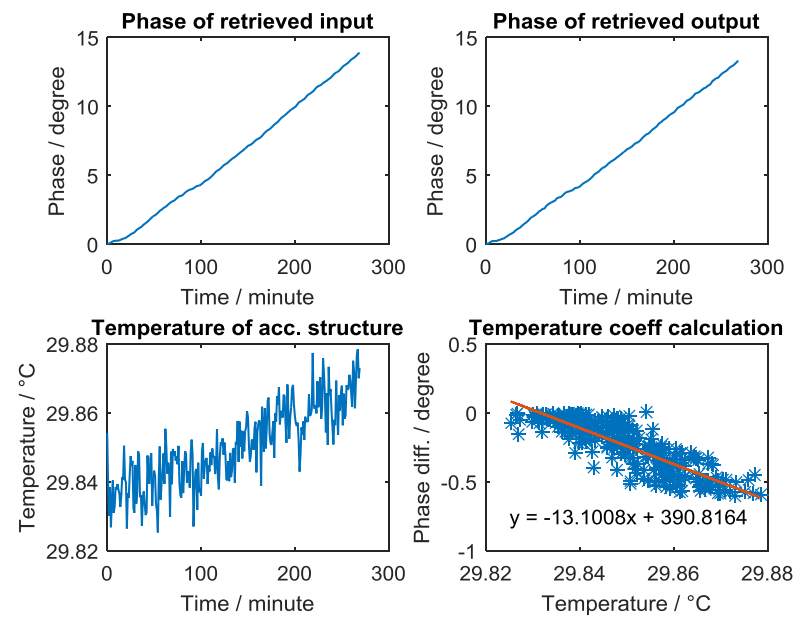

Fig. 17. Correlation between the phase difference and temperature of the first S-band travelling wave structure of the RF station SINSB03 at SwissFEL. The temperature coefficient for phase length drift is about -13.1 degree $/{ }^{\circ} \mathrm{C}$.

\section{LLRF HLA SOFTWARE DESIGN}

The LLRF HLA software was designed in C++ as a module for the Experimental Physics and Industrial Control System (EPICS). The architecture of the software is depicted in Fig. 18.

The major components in the software architecture are summarized in Table II.

The operator or physics applications will execute the procedures and jobs provided by the LLRF HLA via the EPICS database interface. The GUI for the SwissFEL LLRF HLA is depicted in Fig. 19.

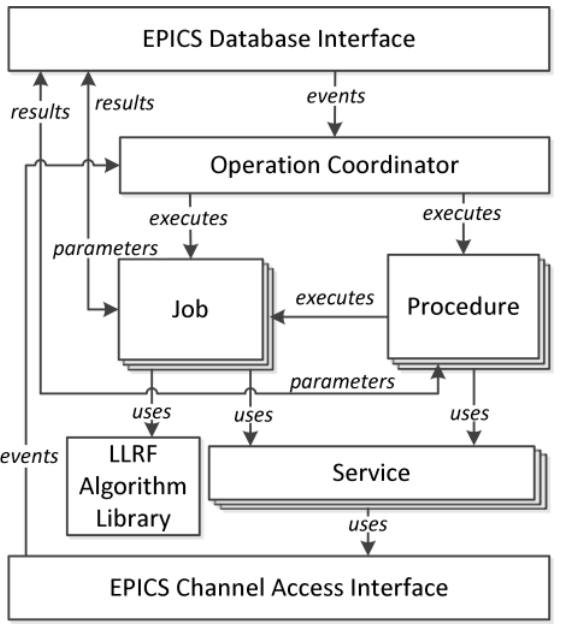

Fig. 18. Architecture of LLRF HLA software.

\begin{tabular}{|c|c|}
\hline Components & Description \\
\hline Coordinator & $\begin{array}{l}\text { An active thread to coordinate the execution of the } \\
\text { procedures and jobs. }\end{array}$ \\
\hline Procedure & $\begin{array}{l}\text { Automation procedure to startup, stop and setup the RF } \\
\text { system. }\end{array}$ \\
\hline Job & A function that the LLRF HLA implements. \\
\hline Service & $\begin{array}{l}\text { An interface to interact with a physical device in the RF } \\
\text { system (e.g. klystron modulator). }\end{array}$ \\
\hline LLRF & A library implemented in $\mathrm{C}$ language to collect \\
\hline Algorithm & algorithms used in LLRF system (e.g. routine to \\
\hline Library & calculate DAC offset). \\
\hline
\end{tabular}

TABLE II

COMPONENTS OF LLRF HLA SOFTWARE

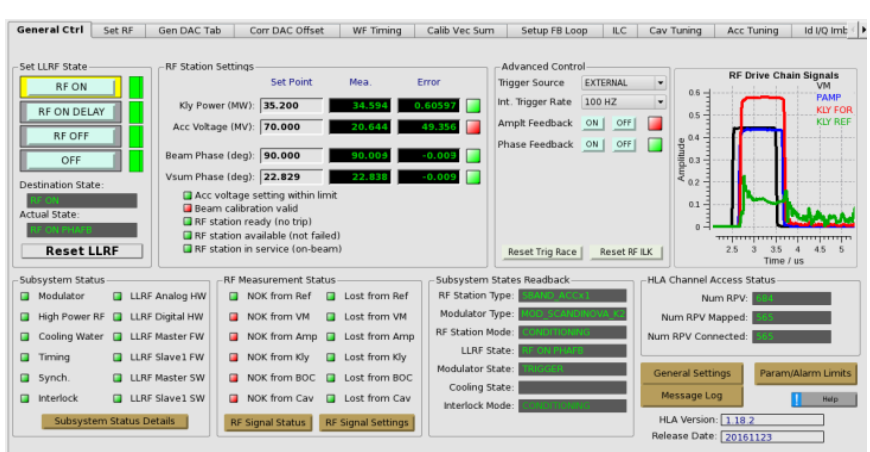

Fig. 19. GUI for SwissFEL LLRF HLA.

\section{CONCLUSION}

The algorithms developed for the LLRF HLA provide practical automation to operate the RF systems of normally conducting Linac. A software package has been well architected and implemented based on EPICS and has been used in daily operation of SwissFEL. The HLA software tools have played an important role in the commissioning of SwissFEL. They have significantly speeded up the settings of the RF stations when applying them on beam acceleration from scratch after high power RF conditioning. The performance optimizations of the RF systems are in progress and the LLRF HLA tools also provide significant help. The software architecture is flexible to add more functions to the HLA. Similar architecture can also be easily applied to other aspects of the accelerator control like the beam based feedback system. The experiences of the design and usage of the LLRF HLA will be also helpful for other accelerator facilities (e.g. European XFEL and LCLS-II) with 
new algorithms for the specific features of their RF systems.

\section{REFERENCES}

[1] V. K. Bharadwaj, K. Bane and J. Clendenin, "Linac Design for the LCLS Project at SLAC," in Proc. PAC'97 conf., Vancouver, BC, Canada, 1997, pp. 903-905.

[2] F. Loehl, J. Alex et al, "Status of the SwissFEL C-Band Linac," in Proc. FEL2014 conf., Basel, Switzerland, 2014, pp. 322-326.

[3] L. Xiao, R.F. Boyce et al, "Dual Feed RF Gun Design for the LCLS," in Proc. PAC'05 conf., Knoxville, Tennessee, USA, 2005, pp. 3432-3434.

[4] U. Ellenberger, H. Blumer et al, "The SwissFEL RF Gun: Manufacturing and Proof of Precision by Field Profile Measurements," in Proc. LINAC2014 Conf., Geneva, Switzerland, 2014, pp. 1117-1119.

[5] Z. Geng and B. Hong, "Design and Calibration of an RF Actuator for Low-Level RF Systems," IEEE Transactions on Nuclear Science, vol. 63, pp. 281-287, Feb. 2016.

[6] Z. Geng et al, "Architecture Design for the SwissFEL LLRF System," in Proc. LINAC2014 Conf., Geneva, Switzerland, 2014, pp. 1114-1116.

[7] A. Hauff et al, "SwissFEL C-Band LLRF Prototype System," in Proc. LINAC2014 Conf., Geneva, Switzerland, 2014, pp. 683-685.

[8] C. Ziomek and P. Corredoura, "Digital I/Q Demodulator," in Proc. PAC'95 Conf., Dallas, Texas, USA, 1995, pp. 2663-2665.

[9] L. Doolittle, "Digital Low-Level RF Control Using Non-IQ Sampling," in Proc. LINAC2006 Conf., Knoxville, Tennessee, USA, 2006, pp. 568-570.

[10] Z.D. Farkas, H.A. Hogg et al, "Recent Progress on SLED, the SLAC Energy Doubler," SLAC-PUB-1561, 1975.

[11] R. Zennaro, M. Bopp et al, "C-Band RF Pulse Compressor for SwissFEL," in Proc. IPAC2013 Conf., Shanghai, China, 2013, pp. 2827-2829.

[12] A. Rezaeizadeh et al, "Model-Based Klystron Linearization in the SwissFEL Test Facility," in Proc. FEL2014 Conf., Basel, Switzerland, 2014, pp. 820-823.

[13] R.K. Jobe, N. Phinney et al, "Phasing Tools for the Klystron at the SLC," in Proc. PAC1989 Conf., Chicago, IL, USA, 1989, pp. 1984-1986.

[14] C. Schmidt et al, "High Performance and Low Latency Single Cavity RF Control Based on MTCA.4," in Proc. ICALEPCS2013 Conf., San Francisco, CA, USA, 2013, pp. 1348-1350.

[15] V. Ayvazyan, S. Choroba, and Z. Geng, "Optimization of Filling Procedure for TESLA-type Cavities for Klystron RF Power Minimization for European XFEL," in Proc. IPAC'10 Conf., Kyoto, Japan, 2010, pp. 1416-1418.

[16] T. Schilcher, "RF Applications in Digital Signal Processing," CERN-2008-003, 2008.

[17] S. Simrock et al, "Universal Controller for Digital RF Control," in Proc. EPAC2006 Conf., Edinburgh, Scotland, 2006, pp. 1459-1461.

[18] W. Cichalewski, "Linearization of Microwave High Power Amplifiers Chain in the RF Systems of Linear Accelerators for FLASH and X-FEL," Ph.D. dissertation, Dept. of Microelectronics and Computer Science, Lodz Univ. of Technology, Lodz, Poland, 2008.

[19] M. Omet, S. Michizono et al, "FPGA-based Klystron Linearization Implementations in Scope of ILC," Nuclear Instruments and Methods in Physics Research A, vol. 768, pp. 69-76, Dec. 2014.

[20] K. Kichhoff, C. Schmidt et al, "An Iterative Learning Algorithm for Control of an Accelerator Based Free Electron Laser," in Proc. 47th IEEE Conf. on Decision and Control, Cancun, Mexico, 2008, pp. 3032-3037.

[21] A. Rezaeizadeh et al, "RF Pulse Flattening in the SwissFEL Test Facility on Model-Free Iterative Learning Control," in Proc. FEL2014 Conf., Basel, Switzerland, 2014, pp. 824-827. 